Research Article

\title{
Combination of Left Ventricular End-Diastolic Diameter and QRS Duration Strongly Predicts Good Response to and Prognosis of Cardiac Resynchronization Therapy
}

\author{
Zhinian Guo, Xiaoyan Liu, Xiaofeng Cheng, Chuan Liu, Ping Li, Yongming He, \\ Rongsheng Rao, Chun Li, Yunlong Chen, Yong Zhang, Xiaoyu Luo, and Jiang Wang \\ Institute of Cardiovascular Diseases, Xinqiao Hospital, Army Medical University (Third Military Medical University), \\ 183 Xinqiao Street, Chongqing 400037, China \\ Correspondence should be addressed to Jiang Wang; 1530579054@qq.com
}

Received 10 October 2019; Accepted 26 December 2019; Published 17 January 2020

Academic Editor: Julian Bostock

Copyright (c) 2020 Zhinian Guo et al. This is an open access article distributed under the Creative Commons Attribution License, which permits unrestricted use, distribution, and reproduction in any medium, provided the original work is properly cited.

\begin{abstract}
Background. Approximately $20-40 \%$ of recipients of cardiac resynchronization therapy (CRT) do not respond to it based on the current patient selection criteria. The purpose of this study was to identify baseline parameters that can predict CRT response and to evaluate the effect of those predictive parameters on long-term prognosis. Methods. This was a retrospective, nonrandomized, noncontrolled cohort study. Patients who received CRT in our centre were divided into responders and nonresponders by the definition of CRT response (an increase in left ventricular ejection fraction (LVEF) of $\geq 5 \%$ and improvement of $\geq 1$ New York Heart Association (NYHA) class from baseline to the 6-month follow-up). Results. Of the 101 patients, 68 were responders and 33 were nonresponders. Left ventricular end-diastolic diameter (LVEDD; OR: 0.88, 95\% CI: 0.81-0.95, $P=0.001$ ) and QRS duration (OR: 1.07, 95\% CI: 1.04-1.10, $P<0.001$ ) were independent predictors of CRT response. The combination of LVEDD and QRS duration was more valuable for predicting CRT response (AUC 0.836; 95\% CI: 0.76-0.91; $P<0.001$ ). Moreover, the combination of LVEDD $\leq 71 \mathrm{~mm}$ and QRS duration $\geq 170 \mathrm{~ms}$ had a low incidence of all-cause mortality, HF hospitalisation, and the composite endpoint. In addition, baseline LVEDD had a positive correlation with QRS duration $(R=0.199, P=0.046)$. Responders to CRT had better LV reverse remodeling. Conclusion. The combination of LVEDD and QRS duration provided more robust prediction of CRT response. Moreover, the combination of LVEDD $\leq 71 \mathrm{~mm}$ and QRS duration $\geq 170 \mathrm{~ms}$ was associated with a low incidence of all-cause mortality, HF hospitalisation, and the composite endpoint. Our results may be useful to provide individualized patient selection for CRT.
\end{abstract}

\section{Introduction}

Cardiac resynchronization therapy (CRT) is an effective therapy for heart failure (HF) patients with reduced left ventricular ejection fraction (LVEF) and intraventricular conduction delay. Large clinical trials have reported that CRT improves cardiac function, HF symptoms, exercise capacity, and quality of life as well as reduces HF-related hospitalizations and decreases mortality [1-4]. Unfortunately, the degree of response to CRT is not the same for all patients. Approximately $20-40 \%$ of patients do not show substantial benefit from CRT with the range depending on the response definition used for "nonresponders" [5]. Therefore, identifying reliable predictors of response prior to CRT implantation using noninvasive tools remains a major challenge faced by researchers. The purpose of the present study was to identify baseline parameters that can predict CRT response at the 6-month follow-up and to evaluate the effect of those predictive parameters on all-cause mortality or HF-related hospitalization. 


\section{Materials and Methods}

2.1. Patient Selection. From January 2014 to December 2018, 118 consecutive patients with congestive HF received CRT. Patients who did not have 6 months of follow-up echocardiography to determine the changes in LVEF were not included in the study $(n=17)$. Hence, a total of 101 consecutive patients were included in the present retrospective, nonrandomized, noncontrolled cohort study. The inclusion criteria were advanced HF of New York Heart Association (NYHA) class II to IV, despite an optimal medical therapy $\geq 3$ months, left ventricular ejection fraction (LVEF) $\leq 35 \%$, and QRS duration $\geq 130 \mathrm{~ms}$. The study was approved by the Clinical Research Ethics Board of Third Military Medical University (Army Medical University). All patients who were familiar with the processes and purposes of the study agreed to participate in this study and signed an informed consent.

2.2. Implantation of CRT. The left ventricular (LV) lead position was selected to achieve satisfactory pacing parameters with no phrenic nerve stimulation. Via the coronary sinus, the LV lead was advanced to the lateral or posterolateral vein. If there was no accessible lateral or posterolateral vein, the great cardiac vein or middle cardiac vein was considered. The right ventricular lead was implanted at the right ventricular apex, and the right atrial lead was placed at the right atrial appendage.

2.3. Echocardiography. All patients underwent echocardiography before CRT implantation and at the 6-month follow-up. Images were obtained using a commercially available system (Vivid 7, General Electric-Vingmed, USA). Echocardiography parameters included LVEF (calculated using modified Simpson's formula), the area of mitral regurgitation (MR; assessed semi-quantitatively), left atrial diameter (LAD), and left ventricular end-diastolic diameter (LVEDD; measured with M-mode).

2.4. Definition of Left Bundle Branch Block (LBBB), Right Bundle Branch Block (RBBB), and CRT Response. LBBB was diagnosed according to conventional criteria, namely a QRS duration $\geq 120 \mathrm{~ms}$ with a QS or rS complex in lead V1 and a monophasic $\mathrm{R}$ wave with no $\mathrm{Q}$ waves in lead V6 $[6,7]$.

$\mathrm{RBBB}$ was defifined as a QRS duration $\geq 120 \mathrm{~ms}$ with a deep terminal S wave in leads I and V6 and an rSR', rsR', or rsr' in lead V1 or V2 $[6,7]$.

The CRT response was defined as the increase of LVEF $\geq 5 \%$ and improvement of $\geq 1$ NYHA class from baseline to the 6-month follow-up $[8,9]$.

2.5. Definition of the Composite Endpoint. The composite endpoint was defined as all-cause mortality or hospitalization for $\mathrm{HF}$.

2.6. Statistical Analysis. Analyses were performed using SPSS version 19.0 (SPSS Inc., USA) and MedCalc version 18.6.0 (MedCalc Inc., Belgium). Continuous variables were presented as the mean \pm standard deviation or median (interquartile range). Categorical variables were presented as numbers with percentages. Differences between parametric variables were evaluated by Student's $t$-test, and differences between nonparametric variables were evaluated by the Mann-Whitney $U$ test. Differences between categorical variables were evaluated by Fisher's exact test or the Chisquare test. Backward stepwise multivariate logistic regression was performed using the variables with $P<0.10$ in the univariate logistic regression. The receiver operating characteristic (ROC) curve was used to visualize the value of variables that could independently predict response in the multivariate analysis, and the optimal cut-off value was defined as the highest level (sensitivity-(1-specificity)). Associations between LVEDD and QRS duration were assessed by linear regression analysis. Kaplan-Meier curves with log-rank tests were generated to assess significant differences in the occurrence of the endpoints. A two-sided $P<0.05$ was considered statistically significant.

\section{Results}

3.1. Baseline Characteristics. Of the 101 patients (mean age of $61.22 \pm 9.54$ years) in the present study, 68 (67.3\%) were considered responders, and $33(32.7 \%)$ were considered nonresponders. The study subjects included 70 (69.3\%) men and 31 (30.7\%) women. Moreover, 62 patients had LBBB, 3 patients had $\mathrm{RBBB}$, and 21 patients had intraventricular conduction delay (IVCD).

As shown in Table 1, responders had significantly smaller LAD $(P=0.009)$, smaller LVEDD $(P=0.010)$, and longer QRS duration $(P<0.001)$ and more frequently suffered from LBBB $(P=0.022)$ than nonresponders. However, there were no significant differences in age, sex, brain natriuretic peptide (BNP), NYHA class, HF duration, hypertension, chronic renal dysfunction (CRD), ischemic cardiomyopathy (ICM), LVEF, MR, and mean follow-up time between the two groups.

3.2. Six-Month Follow-Up. Compared with nonresponders, responders to CRT had greater changes in LVEF, LVEDD, $\mathrm{MR}$, and QRS duration from baseline to the 6-month followup. However, no significant differences in changes in LAD were observed between responders and nonresponders (Table 2).

3.3. Predictors for CRT Response. The backward stepwise multivariate analysis revealed that LVEDD (OR: 0.88, 95\% CI: $0.81-0.95, P=0.001$ ) and QRS duration (OR: $1.07,95 \%$ CI: $1.04-1.10, P<0.001)$ were independent predictors of response (Table 3 ).

ROC curve analysis showed that the area under the curve (AUC) for baseline LVEDD was 0.662 (95\% CI: 0.55-0.77, $P=0.004$ ), with LVEDD $\leq 69 \mathrm{~mm}$ having $62 \%$ sensitivity, $73 \%$ specificity, $82 \%$ positive prediction value (PPV), and $48 \%$ negative prediction value (NPV). For QRS duration, the AUC was 0.744 (95\% CI: $0.65-0.84, P<0.001$ ), with QRS duration $\geq 166 \mathrm{~ms}$ having $62 \%$ sensitivity, $82 \%$ specificity, $88 \% \mathrm{PPV}$, and $51 \%$ NPV. LVEDD $\leq 71 \mathrm{~mm}$ combined with 
TABLE 1: Baseline characteristics.

\begin{tabular}{|c|c|c|c|c|}
\hline Characteristics & Total population $(n=101)$ & Responders $(n=68)$ & Nonresponders $(n=33)$ & $P$ value \\
\hline Age (years) & $61.22 \pm 9.54$ & $61.65 \pm 10.39$ & $60.33 \pm 7.55$ & 0.473 \\
\hline Sex (female) & $31(30.7 \%)$ & $19(27.9 \%)$ & $12(36.4 \%)$ & 0.389 \\
\hline $\mathrm{BNP}(\mathrm{ng} / \mathrm{L})$ & $401(213-1185)$ & $321(181.3-1129)$ & $685(325.5-1230)$ & 0.537 \\
\hline \multicolumn{5}{|l|}{ NYHA class } \\
\hline II & $21(20.8 \%)$ & $15(22.1 \%)$ & $6(18.2 \%)$ & 0.652 \\
\hline III & $60(59.4 \%)$ & $40(58.8 \%)$ & $20(60.6 \%)$ & 0.864 \\
\hline IV & $20(19.8 \%)$ & $13(19.1 \%)$ & $7(21.2 \%)$ & 0.804 \\
\hline HF duration (months) & $48(12-72)$ & $30(12-72)$ & $60(30-96)$ & 0.192 \\
\hline Hypertension, $n(\%)$ & $21(20.8 \%)$ & $15(22.1 \%)$ & $6(18.2 \%)$ & 0.652 \\
\hline CRD, $n(\%)$ & $18(17.8 \%)$ & $11(16.2 \%)$ & $7(21.2 \%)$ & 0.535 \\
\hline $\mathrm{ICM}, n(\%)$ & $17(16.8 \%)$ & $12(17.6 \%)$ & $5(15.2 \%)$ & 0.756 \\
\hline LVEF (\%) & $29.40 \pm 4.42$ & $29.56 \pm 4.10$ & $29.08 \pm 5.06$ & 0.609 \\
\hline $\mathrm{LAD}(\mathrm{mm})$ & $44.39 \pm 5.48$ & $43.41 \pm 5.20$ & $46.43 \pm 5.58$ & 0.009 \\
\hline LVEDD (mm) & $70.06 \pm 7.84$ & $68.68 \pm 7.38$ & $72.91 \pm 8.12$ & 0.010 \\
\hline $\operatorname{MR}\left(\mathrm{cm}^{2}\right)$ & $7.2(4.5-10.4)$ & $7.2(4.5-10.4)$ & $7.5(4.4-10.4)$ & 0.278 \\
\hline QRS duration (ms) & $165.30 \pm 21.33$ & $171.20 \pm 21.56$ & $153.30 \pm 15.07$ & $<0.001$ \\
\hline LBBB, $n(\%)$ & $62(61.4 \%)$ & $47(69.1 \%)$ & $15(45.5 \%)$ & 0.022 \\
\hline Mean follow-up time (months) & $23.76 \pm 14.48$ & $23.60 \pm 14.83$ & $24.09 \pm 13.95$ & 0.875 \\
\hline
\end{tabular}

Values are mean \pm SD, median (range) or $n$ (\%). BNP: brain natriuretic peptide; NYHA: New York Heart Association; HF: heart failure; CRD: chronic renal dysfunction; ICM: ischemic cardiomyopathy; LVEF: left ventricular ejection fraction; LAD: left atrial dimension; LVEDD: left ventricular end-diastolic dimension; MR: mitral regurgitation; LBBB: left bundle branch block.

TABLE 2: Changes in echocardiography and electrocardiogram from baseline to 6 months in two groups.

\begin{tabular}{lccc}
\hline Variables & Responders $(n=68)$ & Nonresponders $(n=33)$ & $P$ value \\
\hline Change in LVEF & $12(8-20)$ & $2[(-4)-4]$ & $<\mathbf{0 . 0 0 1}$ \\
Change in LAD & $-3.2[(-6)-0]$ & $-3.5[(-7)-1.5]$ & 0.649 \\
Change in LVEDD & $-6[(-15)-(-2)]$ & $0[(-3.5)-4.5]$ & $<\mathbf{0 . 0 0 1}$ \\
Change in MR & $-4.5[(-7.2)-(-1.6)]$ & $-2.1[(-5.1)-2.2]$ & $\mathbf{0 . 0 0 8}$ \\
Change in QRS duration & $-34.13 \pm 25.69$ & $-19.26 \pm 24.81$ & $\mathbf{0 . 0 0 9}$ \\
\hline
\end{tabular}

LVEF: left ventricular ejection fraction; LAD: left atrial dimension; LVEDD: left ventricular end-diastolic dimension; MR: mitral regurgitation.

TABLE 3: Univariate and backward stepwise multivariate logistic regression analyses with regard to predictors of response.

\begin{tabular}{lccc}
\hline & \multicolumn{1}{c}{ Univariate analysis } & & Multivariate analysis \\
Baseline characteristics & OR $(95 \% \mathrm{CI})$ & $P$ value & OR (95\% CI) \\
\hline Age & $1.02(0.97-1.06)$ & 0.515 & \\
Sex & $1.47(0.61-3.57)$ & 0.391 & \\
BNP & $1.00(1.00-1.00)$ & 0.533 & \\
NYHA class & $0.86(0.45-1.66)$ & 0.659 & \\
HF duration & $1.00(0.99-1.00)$ & 0.196 & \\
Hypertension & $1.27(0.44-3.25)$ & 0.536 & \\
CRD & $0.72(0.25-2.06)$ & 0.753 & $0.94(0.85-1.04)$ \\
ICM & $1.20(0.39-3.74)$ & 0.605 & $0.88(0.81-0.95)$ \\
LVEF & $1.03(0.93-1.13)$ & $\mathbf{0 . 0 1 2}$ & \\
LAD & $0.90(0.83-0.98)$ & $\mathbf{0 . 0 1 4}$ & $1.07(1.04-1.10)$ \\
LVEDD & $0.93(0.88-0.99)$ & 0.293 & 0.237 \\
MR & $0.96(0.89-1.04)$ & $<\mathbf{0 . 0 0 1}$ & $\mathbf{0 . 0 0 1}$ \\
QRS duration & $1.05(1.03-1.08)$ & $\mathbf{0 . 0 2 4}$ & $<-4.88)$ \\
LBBB & $2.69(1.14-6.33)$ & $0.60-4.001$ \\
\hline
\end{tabular}

OR: odds ratio; 95\% CI: 95\% confidence interval. BNP: brain natriuretic peptide; NYHA: New York Heart Association; HF: heart failure; CRD: chronic renal dysfunction; ICM: ischemic cardiomyopathy; LVEF: left ventricular ejection fraction; LAD: left atrial dimension; LVEDD: left ventricular end-diastolic dimension; MR: mitral regurgitation; LBBB: left bundle branch block.

QRS duration $\geq 170 \mathrm{~ms}$ had $65 \%$ sensitivity, 97\% specificity, 98\% PPV, and 57\% NPV for predicting response (AUC 0.836; 95\% CI: $0.76-0.91 ; P<0.001)$. The combination of LVEDD and QRS duration was more valuable for predicting response than LVEDD (AUC, 0.836 vs. 0.662; $Z=3.058$;
$P=0.002$ ) or QRS duration (AUC, 0.836 vs. 0.744; $Z=2.309 ; P=0.021$ ) alone. However, there was no difference between the value of LVEDD and QRS duration in predicting CRT response (AUC, 0.662 vs. $0.744 ; Z=1.323$; $P=0.186$ ) (Table 4, Figure 1). 
TABLE 4: AUC and cut-off value for LVEDD, QRS duration, and combination of LVEDD and QRS duration.

\begin{tabular}{|c|c|c|c|c|c|c|c|c|}
\hline Variables & AUC & $95 \% \mathrm{CI}$ & $\begin{array}{l}P \text { for } \\
\text { AUC }\end{array}$ & Cut-off value & $\begin{array}{l}\text { Sensitivity } \\
\quad(\%)\end{array}$ & $\begin{array}{l}\text { Specificity } \\
(\%)\end{array}$ & $\begin{array}{r}\text { PPV } \\
(\%)\end{array}$ & $\begin{array}{c}\text { NPV } \\
(\%)\end{array}$ \\
\hline LVEDD (mm) & 0.662 & $0.55-0.77$ & 0.004 & $\leq 69$ & 62 & 73 & 82 & 48 \\
\hline QRS duration (ms) & 0.744 & $0.65-0.84$ & $<0.001$ & $\geq 166$ & 62 & 82 & 88 & 51 \\
\hline $\begin{array}{l}\text { Combination of LVEDD and QRS } \\
\text { duration }(\mathrm{mm}, \mathrm{ms})\end{array}$ & 0.836 & $0.76-0.91$ & $<0.001$ & $\begin{array}{l}\text { LVEDD } \leq 71 \\
\text { and } \geq 170\end{array}$ & 65 & 97 & 98 & 57 \\
\hline
\end{tabular}

LVEDD: left ventricular end-diastolic dimension; AUC: area under the curve; 95\% CI: 95\% confidence interval; PPV: positive prediction value; NPV: negative prediction value.

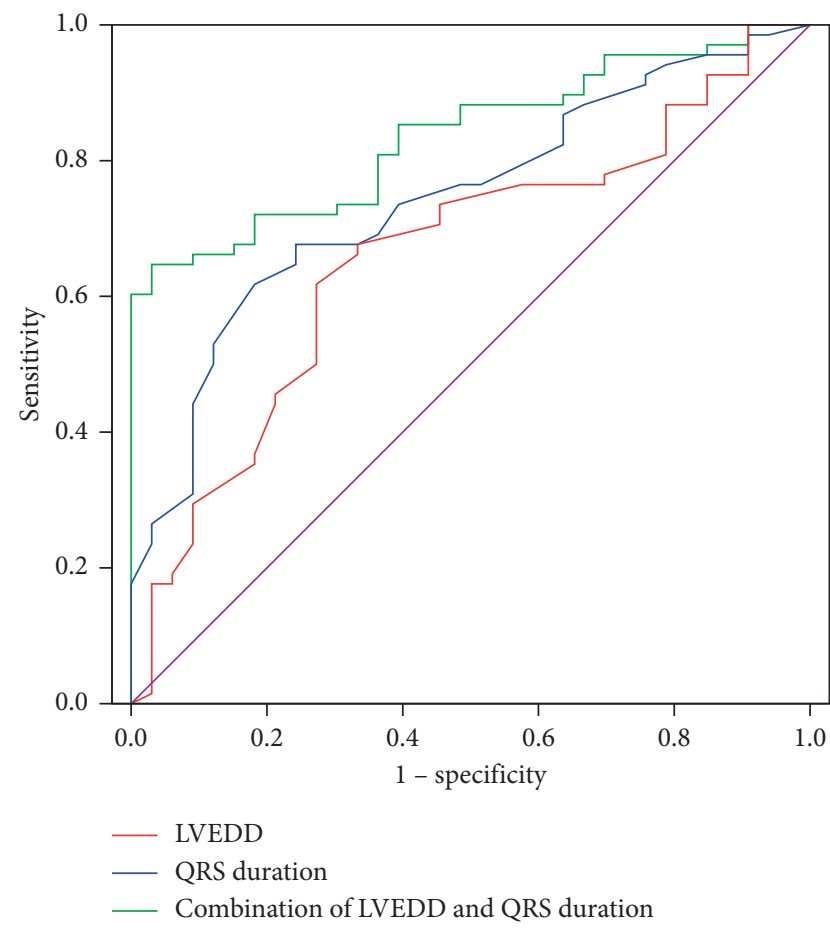

FIGURE 1: Receiver operating characteristic curve for LVEDD (red line), QRS duration (blue line), and combination of LVEDD and QRS duration (green line) in predicting response. Combination of LVEDD and QRS duration versus LVEDD alone AUC (0.836 vs. $0.662, Z=3.058, P=0.002)$. Combination of LVEDD and QRS duration versus QRS duration alone AUC (0.836 vs. 0.744, $Z=2.309, P=0.021)$. LVEDD versus QRS duration AUC (0.662 vs. $0.744, Z=1.323, P=0.186)$. LVEDD: left ventricular end-diastolic dimension; AUC: area under the curve.

In addition, baseline LVEDD had a positive correlation with QRS duration $(R=0.199, P=0.046)$.

3.4. Long-Term Prognosis. Long-term follow-up was performed by telephone interview or clinic visit. During a mean follow-up period of $23.76 \pm 14.48$ months, the composite endpoint occurred in 35 patients (13 deaths and 22 hospitalizations for HF). The incidence of the composite endpoint was $40 \%$ (14 with 4 deaths and 10 hospitalizations for HF) in responders and $60 \%$ in nonrespondents (21 with 9 deaths and 12 hospitalizations). Kaplan-Meier curves showed that the cumulative incidence of the composite endpoint or all-cause mortality alone was significantly lower in responders, patients with LVEDD $\leq 69 \mathrm{~mm}$, and patients with a combination of LVEDD $\leq 71 \mathrm{~mm}$ and QRS duration $\geq 170 \mathrm{~ms}$. Moreover, the endpoint of HF hospitalisation alone was significantly less likely in responders and patients with a combination of LVEDD $\leq 71 \mathrm{~mm}$ and QRS duration $\geq 170 \mathrm{~ms}$. However, no difference was observed between the group with QRS duration $\geq 166 \mathrm{~ms}$ and the group with the QRS duration $<166 \mathrm{~ms}$ for the cumulative incidence of the composite endpoint, all-cause mortality alone or HF hospitalisation alone (Figure 2, Supplementary Figures 1 and 2).

\section{Discussion}

In the present study, we demonstrated that LVEDD and QRS duration were independent predictors of CRT response and that the combination of LVEDD and QRS duration was more effective for response prediction. Moreover, the cumulative incidence of the composite endpoint was statistically lower in responders, LVEDD $\leq 69 \mathrm{~mm}$ patients and combined LVEDD $\leq 71 \mathrm{~mm}$ and QRS duration $\geq 170 \mathrm{~ms}$ patients. Moreover, baseline LVEDD had a positive correlation with QRS duration. In addition, responders to CRT had better LV reverse remodeling.

A previous study by Achilli et al. showed that a smaller LV end-systolic diameter (LVESD) is an independent predictor of a positive response to CRT, with LVESD $<60 \mathrm{~mm}$ having a sensitivity of $66 \%$ and a specificity of $61 \%$ [8]. Goldenberg et al. demonstrated that LV end-diastolic volume (LVEDV) is associated with CRT response [10]. In addition, Rinkuniene et al. reported that LVEDD $<75 \mathrm{~mm}$ is the strongest independent predictor of CRT response [11]. Díaz-Infante et al. also found that LVEDD $\geq 75 \mathrm{~mm}$ is an independent predictor of nonresponse to CRT [12]. In the present study, LVEDD was an independent predictor of CRT response, with $L V E D D \leq 69 \mathrm{~mm}$ showing $62 \%$ sensitivity and $73 \%$ specificity, which was similar to the findings from an earlier study reporting that LVEDD $\leq 67 \mathrm{~mm}$ is associated with CRT response after 6 months of follow-up [13]. Enlarged LV may be a marker of HF progression and impairment of contractile function [8]. Hence, patients with enlarged LVEDD were less likely to respond to CRT [12]. Moreover, Carluccio et al. found that baseline LV endsystolic volume index is a powerful predictor of events (cardiac death and hospital admission for HF) during longterm (40 \pm 23 months) clinical follow-up [14]. A previous study by Adelstein et al. reported that patients with LVEDD $<3.36 \mathrm{~cm} / \mathrm{m}$ height have minimal risk of appropriate shocks after a CRT-D implant [15]. In our study, 


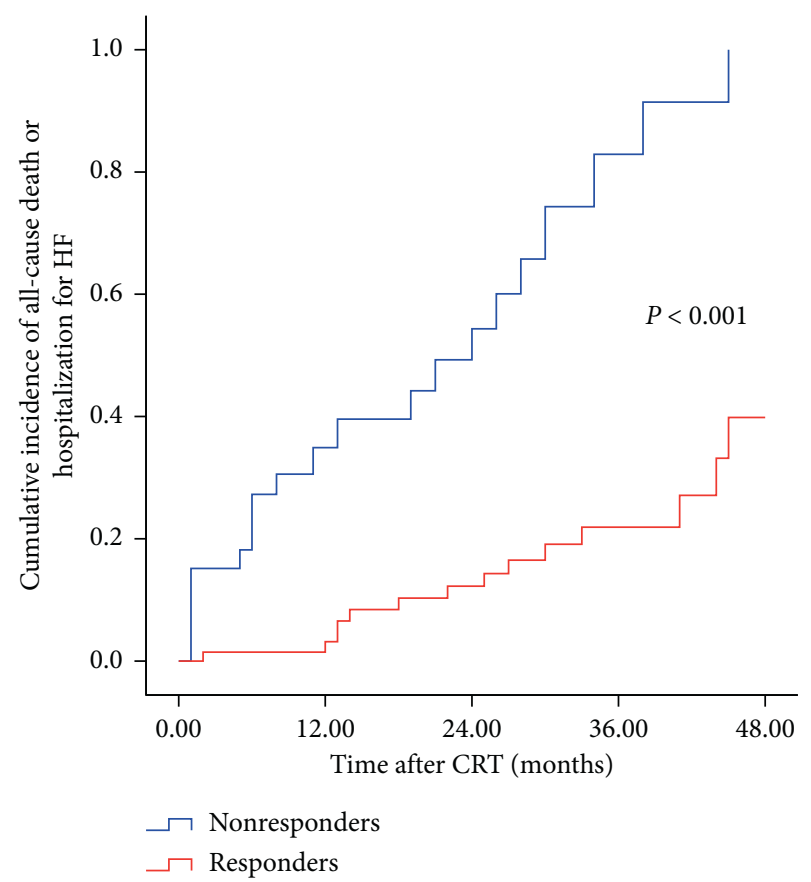

(a)

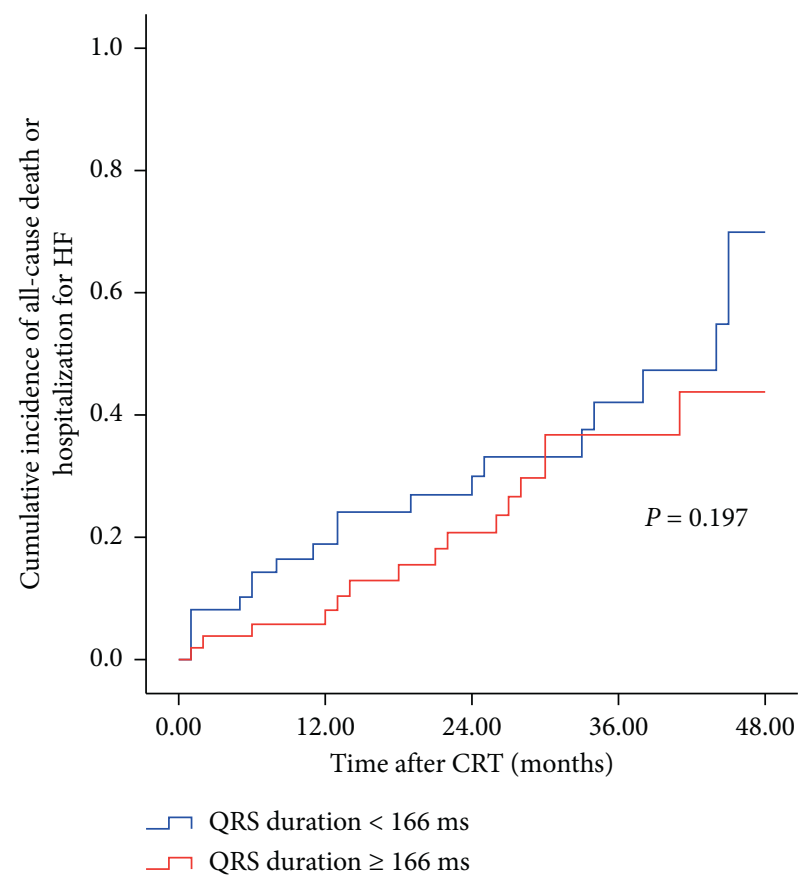

(c)

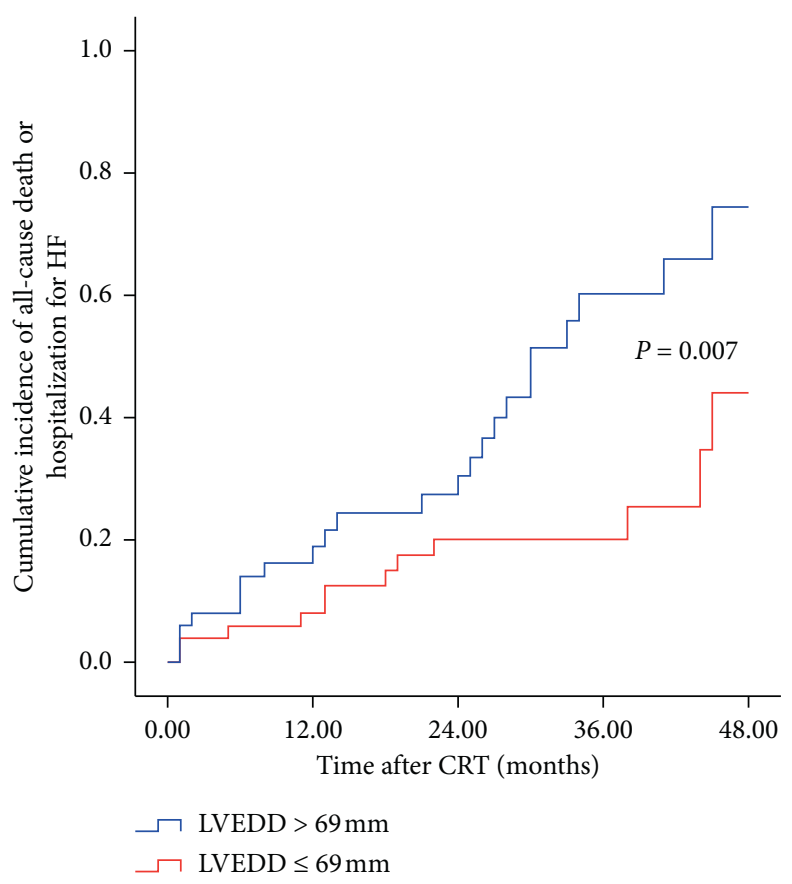

(b)

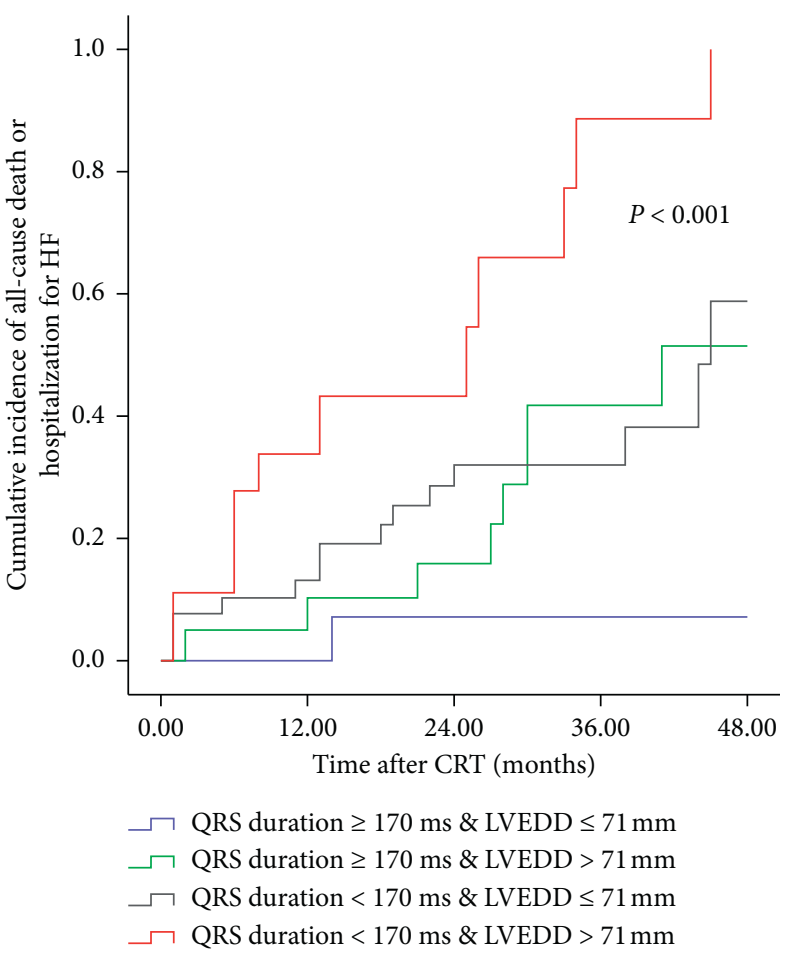

(d)

FIgURE 2: Four Kaplan-Meier curves for response category, LVEDD, QRS duration, and combination of LVEDD and QRS duration of cumulative incidence of composite endpoint at 4 years. (a) For composite endpoint, responders (red line) performed better compared to nonresponders (blue line). (b) For composite endpoint, LVEDD $\leq 69 \mathrm{~mm}$ group (red line) performed better compared to LVEDD $>69 \mathrm{~mm}$ group (blue line). (c) For composite endpoint, there was no difference between QRS duration $\leq 166 \mathrm{~ms}$ group and QRS duration $>166 \mathrm{~ms}$ group. (d) For composite endpoint, combination of QRS duration $\geq 170 \mathrm{~ms}$ and LVEDD $\leq 71 \mathrm{~mm}$ group (blue line) performed the best compared with others. HF: heart failure; LVEDD: left ventricular end-diastolic dimension. 
patients with LVEDD $\leq 69 \mathrm{~mm}$ prior to the CRT implant were associated with a lower risk of the composite endpoint during a mean follow-up period of $23.76 \pm 14.48$ months, which confirmed previous results reporting that larger LV dimensions result in poorer prognoses [14-16].

Wider baseline QRS and a narrowing of the QRS width after CRT implantation are independent predictors of clinical positive response [17]. Another previous study reported that $\mathrm{QRS} \geq 150 \mathrm{~ms}$ is associated with CRT response [10]. The PROSPECT-ECG substudy by Hsing et al. showed that QRS width predicts clinical composite score (CCS) improvement after CRT [18]. Moreover, Linde et al. studied 1591 CRT recipients and reported that QRS duration is a predictor of CRT response and that CRT delivered better benefit to patients with the QRS duration between 160 and $180 \mathrm{~ms}$ [19]. In the present study, the QRS duration $\geq 166 \mathrm{~ms}$ was considered an independent predictor of CRT response, which supported the results above. However, Mollema et al. demonstrated that baseline QRS duration is not predictive of clinical or echocardiographic response to CRT [20]. The inconsistency may be caused by different definitions of CRT response. A meta-analysis of five randomized trials has indicated that QRS duration is a powerful predictor of CRT on morbidity and mortality [21]. However, Leong et al. showed that QRS duration is not associated with death during a median follow-up of 44 months [22]. In our study, compared with patients with a QRS duration $<166 \mathrm{~ms}$, patients with a QRS duration $\geq 166 \mathrm{~ms}$ were not associated with the cumulative incidence of the composite endpoint.

Previous studies have described a positive association between baseline QRS duration and LV size (LV length, LV diameter, and LV mass) [23-26]. Chan et al. showed that LV size increases with prolonged QRS duration in the cardiomyopathy patients [24]. Zweerink et al. reported that the normalization of QRS duration to LV dimension (i.e., QRS duration divided by LV dimension) is associated with CRT response [23]. In line with the previous reports, baseline LVEDD had a positive correlation with QRS duration $(R=0.199, P=0.046)$ in the present study. Rickard et al. reported that there is a weak association between QRS duration and LVEDD $(R=0.106, P<0.001)$ [27]. However, they argued that LVEDD do not modify the effect of QRS duration, by analyzing LVEDD $*$ QRS duration (an interaction term created by Rickard et al.) in the multivariate Cox proportional hazards model [27]. Whether it is scientific and reasonable to analyze $\mathrm{LVEDD} * \mathrm{QRS}$ duration in the multivariate Cox proportional hazards model is warranted to confirm in future studies.

The QRS duration is typically determined by myocardial conduction velocity and conduction path length [23]. LV dilatation may result in increased conduction path length in HF patients. Hence, it is possible that the increase of LV size contributes to the prolongation of the QRS duration [25]. Increased QRS duration was found to be associated with improved CRT response. Nevertheless, progressive LV dilatation limited CRT response. Perhaps, it could be explained by the hypothesis that myocardial conduction velocity rather than conduction path length determines CRT response [23].
In the present study, the combination of LVEDD and QRS duration provided more robust prediction of CRT response than LVEDD or QRS duration alone. Moreover, the combination of LVEDD $\leq 71 \mathrm{~mm}$ and QRS duration $\geq 170 \mathrm{~ms}$ was associated with a low incidence of allcause mortality, HF hospitalisation, or the composite endpoint. Our results may be useful to provide individualized patient selection for CRT. Further investigations are warranted to confirm these results in the future.

Prior studies have reported that LBBB, nonischemic cardiomyopathy (NICM), and female sex seemed to predict CRT response [10, 17, 18, 28, 29]. However, Linde et al. and our study found that they are not associated with CRT response $[19,30]$. Moreover, female patients would have NICM and LBBB more often than male patients [31]. This inconsistency may be due to a great interobserver and intraobserver variability in these parameters.

\section{Limitations}

This study has several limitations. First, the sample size was relatively small. Thus, our results may need to be confirmed by large multicentre prospective studies in the future. Second, our study was a retrospective study, which has inherent potential limitation and may be subject to bias. Third, CRT response was defined at the 6-month follow-up, but complete LVEF recovery after CRT had been reported even after 2 years. Thus, 6-month follow-up may not allow assessment of the time course of LVEF recovery. Finally, patients who were lost at 6-month follow-up were not included in the study, which may result in selection bias.

\section{Conclusions}

The combination of LVEDD and QRS duration provided more robust prediction of CRT response. Moreover, patients with a combination of $L V E D D \leq 71 \mathrm{~mm}$ and QRS duration $\geq 170 \mathrm{~ms}$ had a low incidence of the composite endpoint. Hence, our results may be useful for identifying patients most likely to benefit from CRT.

\section{Data Availability}

The data used to support the findings of this study are available from the corresponding author upon request.

\section{Conflicts of Interest}

The authors declare that they have no conflicts of interest.

\section{Authors' Contributions}

Zhinian Guo and Jiang Wang conceived and designed the study. Zhinian Guo, Xiaoyan Liu, Xiaofeng Cheng, Ping Li, Yongming He, Yunlong Chen, Yong Zhang, and Xiaoyu Luo provided assistance for data acquisition. Rongsheng Rao and Chun Li provided assistance for checking echocardiographic data. Zhinian Guo and Chuan Liu performed the statistical analysis. Zhinian Guo drafted the manuscript, and Jiang Wang revised the manuscript. 


\section{Acknowledgments}

This study was supported by the Clinical Research Fund of Xinqiao Hospital of Third Military Medical University (no. 2014YlC12) and the Starting Fund for Talents Returning from Overseas of Ministry of Education (no. 2015A497).

\section{Supplementary Materials}

Supplemental Figure 1. Four Kaplan-Meier curves for response category, LVEDD, QRS duration, and combination of LVEDD and QRS duration of cumulative incidence of allcause death at 4 years. (A) For cumulative incidence of allcause death, responders (red line) performed better compared with nonresponders (blue line). (B) For cumulative incidence of all-cause death, the LVEDD $\leq 69 \mathrm{~mm}$ group (red line) performed better compared with the LVEDD $>69 \mathrm{~mm}$ group (blue line). (C) For cumulative incidence of all-cause death, there was no difference between the QRS duration $\leq 166 \mathrm{~ms}$ group and QRS duration $>166$ ms group. (D) For cumulative incidence of all-cause death, combination of QRS duration $\geq 170 \mathrm{~ms}$ and the LVEDD $\leq 71 \mathrm{~mm}$ group performed the best compared with others. LVEDD: left ventricular end-diastolic dimension. Supplemental Figure 2. Four Kaplan-Meier curves for response category, LVEDD, QRS duration, and combination of LVEDD and QRS duration of cumulative incidence of hospitalization for heart failure at 4 years. (A) For cumulative incidence of hospitalization for heart failure at 4 years, responders (red line) performed better compared with nonresponders (blue line). (B) For cumulative incidence of hospitalization for heart failure, there was no difference between the LVEDD $\leq 69 \mathrm{~mm}$ group and LVEDD $>69 \mathrm{~mm}$ group (red line). (C) For cumulative incidence of hospitalization for heart failure, there was no difference between the QRS duration $\leq 166 \mathrm{~ms}$ group and QRS duration $>166$ ms group. (D) For cumulative incidence of hospitalization for heart failure, the combination of QRS duration $\geq 170 \mathrm{~ms}$ and LVEDD $\leq 71 \mathrm{~mm}$ group (blue line) performed the best compared with others. LVEDD: left ventricular end-diastolic dimension. . (Supplementary Materials)

\section{References}

[1] C. Linde, S. Mealing, N. Hawkins et al., "Cost-effectiveness of cardiac resynchronization therapy in patients with asymptomatic to mild heart failure: insights from the European cohort of the REVERSE (resynchronization reverses remodeling in systolic left ventricular dysfunction)," European Heart Journal, vol. 32, no. 13, pp. 1631-1639, 2011.

[2] A. S. L. Tang, G. A. Wells, M. Talajic et al., "Cardiacresynchronization therapy for mild-to-moderate heart failure," New England Journal of Medicine, vol. 363, no. 25, pp. 2385-2395, 2010.

[3] A. J. Moss, W. J. Hall, D. S. Cannom et al., "Cardiacresynchronization therapy for the prevention of heart-failure events," New England Journal of Medicine, vol. 361, no. 14, pp. 1329-1338, 2009.
[4] J. G. F. Cleland, J.-C. Daubert, E. Erdmann et al., "The effect of cardiac resynchronization on morbidity and mortality in heart failure," New England Journal of Medicine, vol. 352, no. 15, pp. 1539-1549, 2005.

[5] S. Y. Naqvi, A. Jawaid, I. Goldenberg, and V. Kutyifa, "Nonresponse to cardiac resynchronization therapy," Current Heart Failure Reports, vol. 15, no. 5, pp. 315-321, 2018.

[6] J. L. Willems, E. O. Robles de Medina, R. Bernard et al., "Criteria for intraventricular conduction disturbances and pre-excitation," Journal of the American College of Cardiology, vol. 5, no. 6, pp. 1261-1275, 1985.

[7] B. Surawicz, R. Childers, B. J. Deal, and L. S. Gettes, "AHA/ ACCF/HRS recommendations for the standardization and interpretation of the electrocardiogram," Circulation, vol. 119, no. 10, 2009.

[8] A. Achilli, C. Peraldo, M. Sassara et al., "Prediction of response to cardiac resynchronization therapy: the selection of candidates for CRT (SCART) study," Pacing and Clinical Electrophysiology, vol. 29, no. s2, pp. S11-S19, 2006.

[9] L. A. Pires, W. T. Abraham, J. B. Young, and K. M. Johnson, "Clinical predictors and timing of New York Heart Association class improvement with cardiac resynchronization therapy in patients with advanced chronic heart failure: results from the multicenter insync randomized clinical evaluation (MIRACLE) and multicenter inSync ICD randomized clinical evaluation (MIRACLE-ICD) trials," American Heart Journal, vol. 151, no. 4, pp. 837-843, 2006.

[10] I. Goldenberg, A. J. Moss, W. J. Hall et al., "Predictors of response to cardiac resynchronization therapy in the multicenter automatic defibrillator implantation trial with cardiac resynchronization therapy (MADIT-CRT)," Circulation, vol. 124, no. 14, pp. 1527-1536, 2011.

[11] D. Rinkuniene, S. Bucyte, K. Ceseviciute et al., "Predictors of positive response to cardiac resynchronization therapy," BMC Cardiovascular Disorders, vol. 14, no. 1, p. 55, 2014.

[12] E. Díaz-Infante, L. Mont, J. Leal et al., "Predictors of lack of response to resynchronization therapy," The American Journal of Cardiology, vol. 95, no. 12, pp. 1436-1440, 2005.

[13] S. Buck, A. H. Maass, W. Nieuwland, R. L. Anthonio, D. J. Van Veldhuisen, and I. C. Van Gelder, "Impact of interventricular lead distance and the decrease in septal-tolateral delay on response to cardiac resynchronization therapy," Europace, vol. 10, no. 11, pp. 1313-1319, 2008.

[14] E. Carluccio, P. Biagioli, G. Alunni et al., "Presence of extensive LV remodeling limits the benefits of CRT in patients with intraventricular dyssynchrony," JACC: Cardiovascular Imaging, vol. 4, no. 10, pp. 1067-1076, 2011.

[15] E. C. Adelstein, D. Schwartzman, S. Jain, R. Bazaz, N. C. Wang, and S. Saba, "Left ventricular dimensions predict risk of appropriate shocks but not mortality in cardiac resynchronization therapy-defibrillator recipients with left bundle-branch block and non-ischemic cardiomyopathy," EP Europace, vol. 19, no. 10, pp. 1689-1694, 2017.

[16] H. D. White, R. M. Norris, M. A. Brown, P. W. Brandt, R. M. Whitlock, and C. J. Wild, "Left ventricular end-systolic volume as the major determinant of survival after recovery from myocardial infarction," Circulation, vol. 76, no. 1, pp. 44-51, 1987.

[17] S. Yeim, P. Bordachar, S. Reuter et al., "Predictors of a positive response to biventricular pacing in patients with severe heart failure and ventricular conduction delay," Pacing and Clinical Electrophysiology, vol. 30, no. 8, pp. 970-975, 2007. 
[18] J. M. Hsing, K. A. Selzman, C. Leclercq et al., "Paced left ventricular QRS width and ECG parameters predict outcomes after cardiac resynchronization therapy: PROSPECT-ECG substudy," Circulation: Arrhythmia and Electrophysiology, vol. 4, no. 6, pp. 851-857, 2011.

[19] C. Linde, W. T. Abraham, M. R. Gold et al., "Predictors of short-term clinical response to cardiac resynchronization therapy," European Journal of Heart Failure, vol. 19, no. 8, pp. 1056-1063, 2017.

[20] S. A. Mollema, G. B. Bleeker, E. E. van der Wall, M. J. Schalij, and J. J. Bax, "Usefulness of QRS duration to predict response to cardiac resynchronization therapy in patients with endstage heart failure," The American Journal of Cardiology, vol. 100, no. 11, pp. 1665-1670, 2007.

[21] J. G. Cleland, W. T. Abraham, C. Linde et al., "An individual patient meta-analysis of five randomized trials assessing the effects of cardiac resynchronization therapy on morbidity and mortality in patients with symptomatic heart failure," European Heart Journal, vol. 34, no. 46, pp. 3547-3556, 2013.

[22] D. P. Leong, U. Höke, V. Delgado et al., "Right ventricular function and survival following cardiac resynchronisation therapy," Heart, vol. 99, no. 10, pp. 722-728, 2013.

[23] A. Zweerink, L. Wu, G. J. de Roest et al., "Improved patient selection for cardiac resynchronization therapy by normalization of QRS duration to left ventricular dimension," $E P$ Europace, vol. 19, no. 9, pp. 1508-1513, 2016.

[24] D. D. Chan, K. C. Wu, Z. Loring et al., "Comparison of the relation between left ventricular anatomy and QRS duration in patients with cardiomyopathy with versus without left bundle branch block," The American Journal of Cardiology, vol. 113, no. 10, pp. 1717-1722, 2014.

[25] R. A. Stewart, A. A. Young, C. Anderson, K. K. Teo, G. Jennings, and B. R. Cowan, "Relationship between QRS duration and left ventricular mass and volume in patients at high cardiovascular risk," Heart, vol. 97, no. 21, pp. 17661770, 2011.

[26] O. De Winter, N. Van de Veire, F. Van Heuverswijn, G. Van Pottelberge, T. C. Gillebert, and J. De Sutter, "Relationship between QRS duration, left ventricular volumes and prevalence of nonviability in patients with coronary artery disease and severe left ventricular dysfunction," European Journal of Heart Failure, vol. 8, no. 3, pp. 275-277, 2006.

[27] J. Rickard, B. Baranowski, R. A. Grimm et al., "Left ventricular size does not modify the effect of QRS duration in predicting response to cardiac resynchronization therapy," Pacing and Clinical Electrophysiology, vol. 40, no. 5, pp. 482-487, 2017.

[28] J. Rickard, H. Michtalik, R. Sharma et al., "Predictors of response to cardiac resynchronization therapy: a systematic review," International Journal of Cardiology, vol. 225, pp. 345-352, 2016.

[29] T. P. Cappola, M. R. Harsch, M. Jessup et al., "Predictors of remodeling in the CRT era: influence of mitral regurgitation, BNP, and gender," Journal of Cardiac Failure, vol. 12, no. 3, pp. 182-188, 2006.

[30] E. S. Chung, A. R. Leon, L. Tavazzi et al., "Results of the predictors of response to CRT (PROSPECT) trial," Circulation, vol. 117, no. 20, pp. 2608-2616, 2008.

[31] Y.-Z. Xu, P. A. Friedman, T. Webster et al., "Cardiac resynchronization therapy: do women benefit more than men?," Journal of Cardiovascular Electrophysiology, vol. 23, no. 2, pp. 172-178, 2012. 


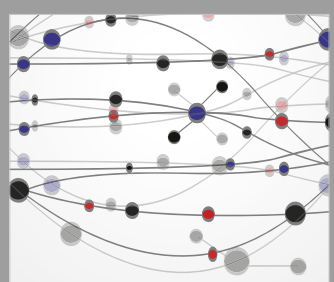

The Scientific World Journal
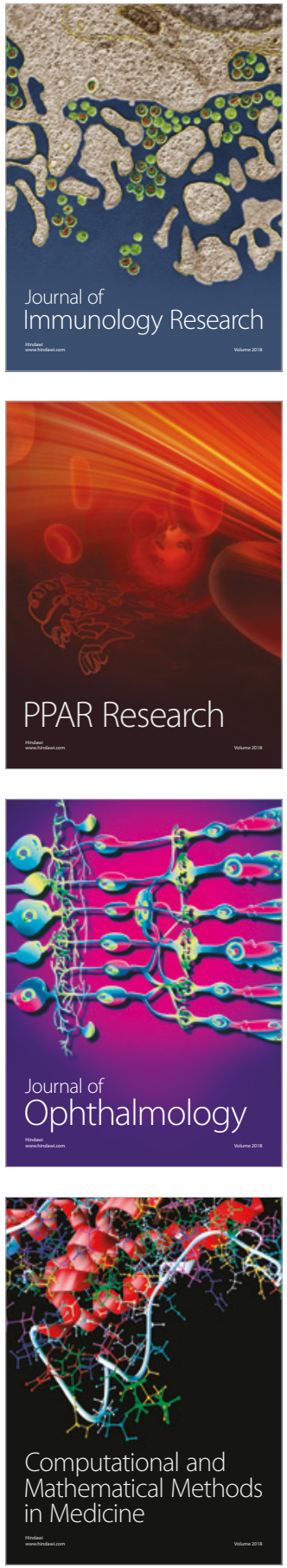

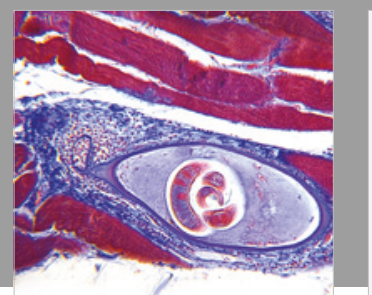

Gastroenterology Research and Practice

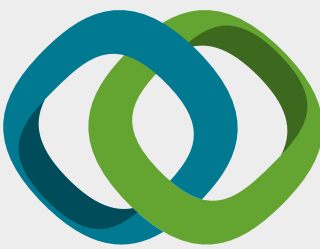

\section{Hindawi}

Submit your manuscripts at

www.hindawi.com
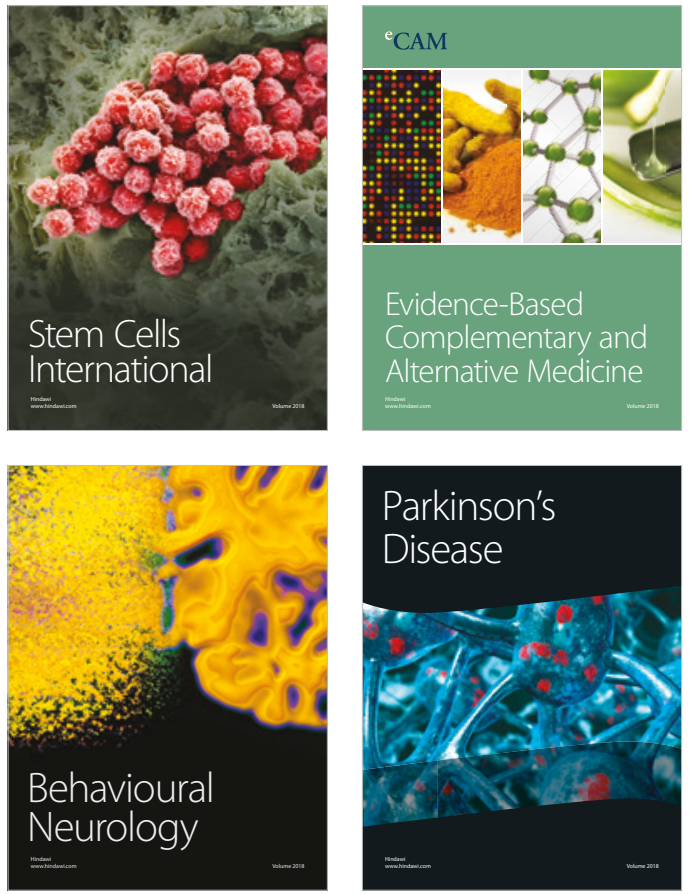

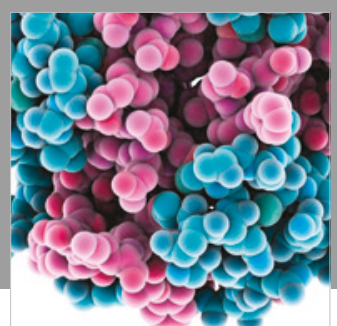

ournal of

Diabetes Research

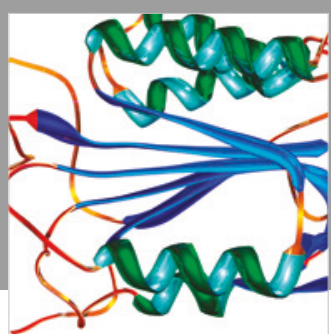

Disease Markers
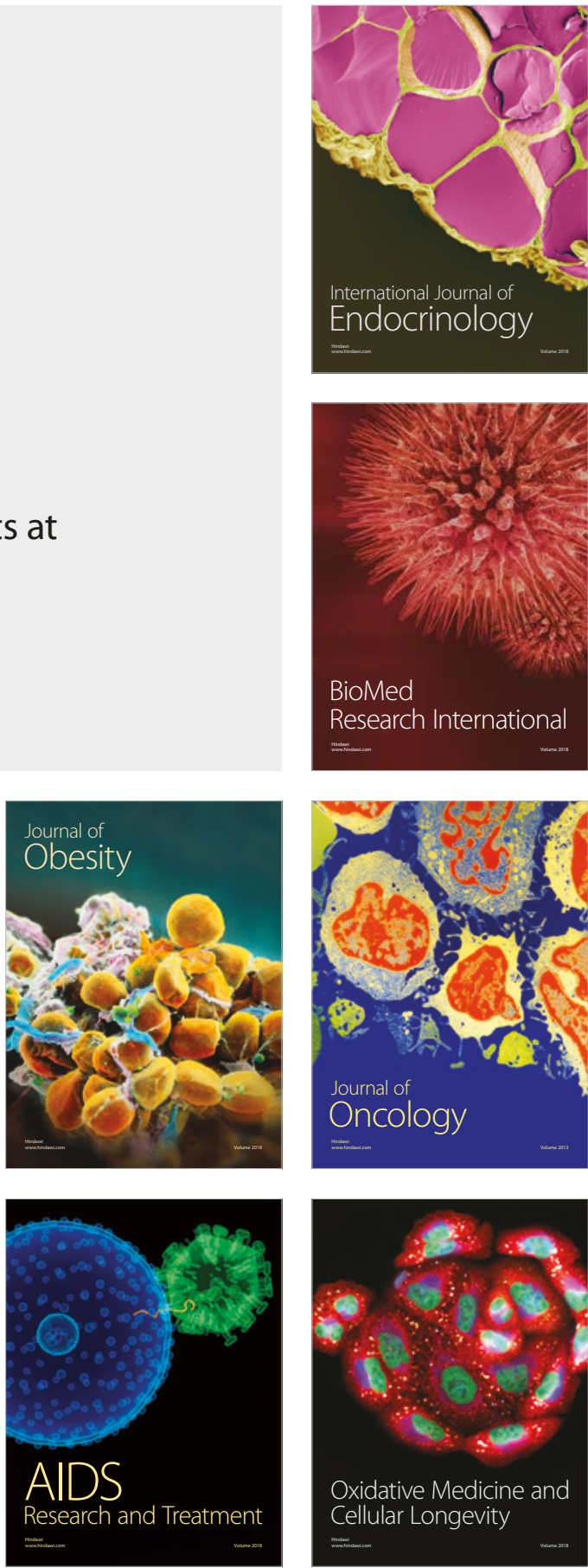